ARTÍCULO DE INVESTIGACIÓN

\title{
Teledetección satelital cuantitativa para estimar el área basal del bosque de Nothofagus pumilio (Nothofagaceae): El rol del índice de área foliar como información auxiliar
}

\author{
Quantitative remote sensing to estimate basal area in Nothofagus pumilio
}

(Nothofagaceae) forest: The role of leaf area index as ancillary information

\author{
GASTÓN M. DÍAZ ${ }^{1,2, *}$, DIEGO MOHR-BELL ${ }^{1}$, JOSÉ D. LENCINAS1, \\ MARCOS MENGER ${ }^{3} \&$ HÉCTOR F. DEL VALLE $^{2,4}$
}

\author{
${ }^{1}$ Centro de Investigación y Extensión Forestal Andino Patagónico (CIEFAP), ruta 259 km 4 (9200) Esquel, Chubut, Argentina \\ ${ }^{2}$ Consejo Nacional de Investigaciones Científicas y Técnicas (CONICET), Argentina \\ ${ }^{3}$ Dirección general de boques y parques de Chubut, Argentina \\ ${ }^{4}$ Centro Nacional Patagónico (CENPAT-CONICET), Puerto Madryn, Chubut, Argentina \\ *Autor correspondiente: gdiaz@ciefap.org.ar
}

\begin{abstract}
RESUMEN
Los bosques de lenga (Nothofagus pumilio) son el recurso forestal más importante de la región andino patagónica argentina, sin embargo, para implementar planes de manejo en pos de prevenir o revertir su degradación es necesario disponer de mayor información sobre su estructura. Una alternativa para obtener esa información es relacionar datos satelitales con las características del bosque a través de modelos físicos y estadísticos. Pero, ¿cuál es el método más eficaz? El índice de área foliar (IAF) se encuentra relacionado con la reflectividad del dosel vegetal a través del modelo de transferencia radiativa PROSAIL, lo que permite desarrollar técnicas de teledetección satelital para estimar el IAF con bases físicas, en vez de con modelos estadísticos. De ese modo se puede aprovechar la relación empírico-biológica existente entre el IAF y la estructura del bosque para estimar el área basal. El objetivo principal fue comparar la exactitud de tres métodos para estimar el área basal de la lenga con datos SPOT-5. Los métodos comparados fueron: estadístico directo (ED), estadístico de dos pasos (E2P) y físico-estadístico de dos pasos (FE2P). La exactitud fue evaluada con 24 parcelas no involucradas en el ajuste o calibración de los modelos. No se hallaron diferencias significativas en la exactitud de la alternativa físico-estadística en comparación con las netamente estadísticas. Sin embargo, en base al análisis conjunto de los resultados y lo reportado por otros investigadores, se concluye que la ventaja de utilizar modelos físicos radica en la mayor robustez de la estimación y no en la mayor exactitud.
\end{abstract}

Palabras clave: imagen satelital de alta resolución, inventario forestal, NDVI, PROSPECT, SAIL.

\begin{abstract}
Lenga forests (Nothofagus pumilio) are the most important forest resource of the Argentinean Andean Patagonia, however, more information about their structure is needed to implement forest management policies and practices in order to prevent their degradation and revert it. One option to obtain this kind of information is to relate satellite data to the forest characteristics. Nevertheless, which is the most efficient estimation methodology? Leaf area index (LAI) is related to the canopy reflectance through radiative transfer model PROSAIL, allowing the development of LAI physical based estimation techniques, instead of statistical models. Therefore, the empirical-biological relationship between LAI and the forest structure can be used to estimate structural parameters, as basal area. The aim of this study was to compare the accuracy of three different approaches to estimate basal area using SPOT-5 data. The approaches compared were: direct statistics (ED), two steps statistics (E2P), and two steps physics-statistics (FE2P). Accuracy assessment was done with 24 independent field measurements. The difference in accuracy was not statistically significant, i.e., the physical-statistical model was not more accurate than the purely statistical model. However, based on analysis of other authors evidence and the results of this study, the conclusion was that the advantage of using physical model, lies on the greater robustness, and not on the better accuracy.
\end{abstract}

Key words: forest inventory, high resolution satellite imagery, NDVI, PROSPECT, SAIL. 


\section{INTRODUCCIÓN}

En la región cordillerana de Patagonia Argentina, los bosques de lenga (Nothofagus pumilio [Poepp. et. Endl.] Krasser) desempeñan importantes funciones ecológicas $\mathrm{y}$ prestan valiosos servicios sociales por su elevado valor económico y paisajístico, destacándose su rol en la protección de las principales cuencas colectoras de agua (Veblen 1996, Martínez-Pastur 2000, Bava \& Lopez-Bernal 2005). La lenga es la especie que cubre la mayor extensión dentro de la región fitogeográfica del bosque andino patagónico (SAyDS 2005). Esta región, en el contexto de la fijación de carbono atmosférico, reviste especial importancia porque reserva un cuarto del carbono almacenado en el bosque nativo argentino, aunque corresponde solo al $6 \%$ de su superficie total (SAyDS 2005, Alcobé 2008). Por lo tanto, la conservación y el manejo sustentable de la lenga, para mantener e incrementar su biomasa acumulada, es una contribución de interés para reducir, a nivel mundial, la emisión de gases de efecto invernadero debido a la deforestación y la degradación forestal (Naciones Unidas 2009, Pelletier et al. en prensa).

Desde el punto de vista de la fijación de carbono atmosférico, el índice de área foliar (IAF) es de importancia por su rol en la dinámica de intercambio con la atmósfera (Bréda 2003). El IAF es el cociente entre la mitad de la superficie total de los tejidos fotosintéticos de un área determinada de vegetación y la superficie horizontal que esta cubre (Lang 1991, Chen \& Black 1992, originalmente definido por Watson 1947). Sin embargo, para implementar planes de manejo en pos de detener la degradación y aumentar la biomasa acumulada, se requiere conocer otros parámetros biofísicos útiles para el manejo (Martínez-Pastur et al. 2010) y no solamente el IAF.

Una alternativa para estimar parámetros biofísicos (IAF $\mathrm{u}$ otros habitualmente medidos en inventarios forestales) es la teledetección satelital cuantitativa, cuyos métodos son tradicionalmente divididos en estadísticos y físicos (Liang 2007). En boques de lenga solamente se han utilizado métodos de relación estadística directa, los cuales consisten en realizar la estimación en un solo paso bajo el supuesto de una buena correlación estadística entre los datos satelitales y la variable de interés (Böswald et al. 2002, Eckert et al. 2005, Poulain et al. 2010).

Los datos satelitales ópticos registran, principalmente, la respuesta espectral de la parte superior de las copas y los claros del bosque. Si la variable de interés no puede ser detectada considerando esa limitación, en realidad se está aprovechando la correlación entre la variable de interés y una variable detectable (Fassnacht et al. 2006). Hall et al. (2006) hacen explícito ese mecanismo y proponen un método estadístico de dos pasos para estimar parámetros biofísicos de bosques en Canadá. Siendo el primer paso estimar variables auxiliares estrechamente relacionadas con los datos satelitales; y el segundo paso, utilizar esa estimación para calcular los parámetros biofísicos habitualmente medidos en inventarios forestales.

El uso del IAF como variable auxiliar en una estimación de dos pasos es promisorio, tanto si se considera el fundamento físico que lo relaciona con los datos satelitales ópticos (Verhoef 1984), como si se consideran las evidencias reunidas que verifican su alta relación con ellos (Fassnacht 1997, Chen 2002, Eklundh et al. 2003, Hall et al. 2003a, 2003b, Fernandes et al. 2004, Lee et al. 2004, Tan et al. 2006, Flores et al. 2006, Yang et al. 2006, Houborg et al. 2007, Sprintsin et al. 2007, Fang et al. 2008, Shin et al. 2010). Además de ser de interés por sí mismo, el IAF tiene la ventaja adicional de poder estimarse con modelos físicos. Dentro de ese tipo de modelos, el PROSAIL es uno de los más difundidos. Este permite describir la variación espectral y direccional de la reflectividad en función de la estructura-bioquímica de las hojas y la estructura del dosel (Jacquemoud et al. 2009). Es posible que al utilizar un método físico se alcancen resultados más exactos, aunque su principal ventaja reside en la robustez y no en la exactitud (Yebra et al. 2008b). Esto es porque, a diferencia de los modelos estadísticos, los modelos físicos no son dependientes de los datos usados en la calibración y de las condiciones bajo las cuales estos fueron tomados, característica que les otorga mayor poder de generalización (Yebra et al. 2008a). Sin embargo, su desventaja reside 
en lo complejo de su utilización (Liang 2003, capítulo 1).

Los parámetros biofísicos pueden clasificarse en de primer orden y de segundo orden (Gemmell \& Varjo 1999, Soenen et al. 2010), diferenciándose en que solamente los primeros pueden obtenerse directamente de la inversión de modelos físicos. Considerando que la mayoría de los parámetros habitualmente medidos en inventario forestal son de segundo orden, estos deberían ser obtenidos en un segundo paso a través de su relación con los de primer orden. Por lo tanto, podría modificarse la propuesta de Hall et al. (2006) y reemplazar el primer paso, originalmente estadístico, por la inversión del PROSAIL para estimar el IAF. De esta manera, el IAF cumpliría el rol de variable auxiliar (parámetro biofísico de primer orden).

Un inventario forestal debe tener como escala objetivo el nivel de rodal si su fin es aportar información útil para establecer pautas de manejo sustentable (Bettinger et al. 2008, capítulo 3). Para ello, el satélite SPOT-5 (del francés Système pour l'Observation de la Terre $5)$, con un sensor multiespectral de $10 \mathrm{~m}$ de resolución espacial, es una alternativa razonable en términos de costo-beneficio (Lencinas 2009, Wolter et al. 2009).

Este estudio aborda la pregunta ¿cuál es el método más eficaz para estimar parámetros biofísicos del bosque de lenga con datos satelitales ópticos? El objetivo principal es comparar la exactitud de tres métodos para estimar el área basal de la lenga con datos SPOT-5. Los métodos a comparar son: estadístico directo (ED), estadístico de dos pasos (E2P) y físico-estadístico de dos pasos (FE2P). El objetivo secundario es desarrollar un método simplificado para invertir el PROSAIL con datos satelitales y estimar el IAF. La hipótesis es: para estimar el área basal de la lenga con datos satelitales ópticos es más efectivo, en vez de emplear una aproximación solo estadística, utilizar un método físicoestadístico. La predicción asociada a esta hipótesis es que el método FE2P será, al menos, de exactitud equivalente a los métodos estadísticos puros. Esto implicaría que el método físico-estadístico sería más efectivo que los métodos puramente estadísticos porque, con igual exactitud, aportaría mayor robustez.

\section{MÉTODOS}

\section{Área de estudio}

El área de estudio ( $43^{\circ} 50^{\prime} \mathrm{S}$ y $\left.71^{\circ} 50^{\prime} \mathrm{O}\right)$ tiene una extensión de $25 \mathrm{~km}^{2}$ (Fig. 1) e incluye la reserva forestal "Lago Guacho" (Chubut, Argentina). Las especies arbóreas del lugar son la lenga y el ñire (Nothofagus antarctica [G. Forster] Oerst), la primera es predominante y se puede encontrar en todas sus fases de desarrollo, la segunda está asociada a zonas de menor altura, a suelos saturados de agua (mallines) o a suelos de poca profundidad. La lenga del área es de estructura irregular debido al tipo de interacción entre la dinámica de regeneración y la dinámica de disturbios. La lenga forma un banco de plántulas que responde a la liberación de recursos por apertura de claros (Martínez-Pastur et al. 2007). Estos pueden deberse a la caída de árboles individuales o de grandes grupos, como las producidas por fuertes vientos (Veblen 1996). Estas dos alternativas de disturbio son, en realidad, los extremos de un continuo. La primera condiciona estructuras irregulares y se conoce como dinámica de claros; la segunda, asociada a grandes disturbios, condiciona estructuras coetáneas y regulares (Bava \& Rechene 2004, Veblen et al. 2004

\section{Estimación del IAF en el terreno}

El IAF fue calculado con fotografías hemisféricas (Bréda 2003) tomadas con la cámara digital Nikon 5700 con un convertidor Nikon FC-E9. El equipo fue montado en una base niveladora para que el centro de la fotografía coincida con el cenit. Las fotografías fueron almacenadas en formato JPG de máxima calidad. Cada imagen tuvo un diámetro de 1555 píxeles desde 0 a $90^{\circ}$ de ángulo cenital. Las fotografías se tomaron en días completamente nublados y con la exposición en modo automático. El software GIMP 2.6.11 (http://www.gimp.org) se utilizó para segmentar cada fotografía mediante un valor umbral determinado manualmente (Jonckheere et al. 2005). Las fotografías segmentadas fueron procesadas con el Hemisfer 1.5.0 (C) Patrick Schleppi, WSL Birmensdorf, $\mathrm{CH})$ configurado para dividir la imagen en tres anillos de $15^{\circ}$ de ángulo cenital (Thimonier et al. 2010) y calcular el IAF efectivo con el algoritmo de Lang (1987).

\section{Diseño de muestreo}

En el área de estudio, los bosques con menor densidad de copa cubren menos superficie. Para procurar una muestra representativa del gradiente de cobertura de copas, fue utilizado un diseño de muestreo aleatorio estratificado (Kangas \& Maltamo 2006). Los estratos establecidos fueron: (A) cobertura continua de copas con estructura vertical homogénea; (B) cobertura continua de copas con estructura vertical heterogénea; (C) cobertura discontinua de copas con claros de cinco a veinte metros de radio y (D) cobertura discontinua de copas con alta frecuencia espacial de claros mayores a $15 \mathrm{~m}$ de radio (el método de estratificación se desarrolla en Material Complementario). En cada estrato se distribuyeron 11 unidades de muestreo, cada una consistió en dos parcelas circulares concéntricas de 500 y $300 \mathrm{~m}^{2}$. En la primera fueron medidos los árboles mayores a $30 \mathrm{~cm}$ de diámetro a la altura del pecho; en la segunda, los mayores a diez y menores o iguales a $30 \mathrm{~cm}$. Los datos fueron procesados en gabinete para obtener el área basal por hectárea. Se tomaron cinco fotografías hemisféricas en cinco parcelas de cada 
estrato; la primera fue tomada en el centro de la parcela, las restantes a ocho metros desde la posición anterior y hacia los cuatro puntos cardinales.

\section{Geomática: Geoposicionamiento y procesamiento de datos satelitales}

Para medir coordenadas geográficas en el terreno fueron empleados los equipos GPS Trimble GeoXT y 4600SL, utilizándose el método estático-rápido con corrección diferencial (Trimble 2001). Las mediciones se realizaron en fase cuando se trató de puntos de control en el terreno para la corrección geométrica de los datos satelitales (GCP, del inglés Ground Control Point); en cambio, durante la localización de las unidades de muestreo dentro del bosque, las mediciones debieron realizarse en código, principalmente a causa de la interferencia del dosel forestal.

Se utilizaron datos SPOT-5 adquiridos en verano el 02/02/2007, sin nubes sobre el área de estudio y con un ángulo fuera del nadir de $17.7^{\circ}$. El sensor tiene cuatro bandas de 8 bit en el verde, rojo, infrarrojo cercano e infrarrojo medio, con resolución espacial de $10 \mathrm{~m}$, salvo por la banda en el infrarrojo medio, que es adquirida a 20 $\mathrm{m}$ y, mediante remuestreo bilineal (M Travers - soporte técnico al cliente de Spot Image, comunicación personal, 2011), distribuida con $10 \mathrm{~m}$.

Los datos fueron solicitados a Spot Image (http:// www.spotimage.com) con el nivel de procesamiento $1 \mathrm{~A}$ DIMAP, en el que se corrigen los errores radiométricos originados en las diferencias de sensibilidad entre los detectores elementales del sensor (Spot Image 2010). Los datos fueron ortorrectificados con el software Geomática PCI 10.1.3, utilizando el modelo físico de
Toutin, 31 GCP y un modelo digital de elevación con exactitud altimétrica de 16 m de RMS (del inglés Root Mean Square). Para generar la ortoimagen se utilizó como método de remuestreo el del vecino más próximo. Como resultado, la exactitud planimétrica fue de $21 \mathrm{~m}$ de RMS. Los datos fueron calibrados y corregidos por los efectos de la atmósfera y del relieve mediante el modelo ATCOR-3 del software ATCOR-2/3 7.1. Todos los procedimientos fueron realizados en el sistema de coordenadas Gauss-Krüger faja 1 con datum y esferoide WGS84. Para facilitar la interpretación y comparación con sistemas internacionales, se denominó $\mathrm{x}$ al eje en sentido oeste-este (para mayor detalle, véase el Material Complementario).

Para asociar los datos SPOT-5 con la información obtenida en el terreno, se calculó el índice de vegetación de diferencia normalizada (NDVI) con la reflectividad de superficie de las bandas del rojo y el infrarrojo cercano. Con una ventana de $3 \times 3$ píxeles se obtuvo la mediana del NDVI, siendo el píxel central de cada ventana coincidente con la posición tomada en el terreno con el GPS (Hall et al. 2006, Labrecque et al. 2006, Poulain et al. 2010).

PROSAIL: Descripción del modelo, método de inversión y método de calibración

El PROSAIL es la conjunción de los modelos PROSPECT y SAIL, de modo que la salida del primero sirve de entrada al segundo. Para su cálculo se suponen las siguientes simplificaciones: el dosel es horizontal y se extiende infinitamente, su único componente son las hojas y su distribución es homogénea. A pesar de estas grandes simplificaciones, el PROSAIL ha sido

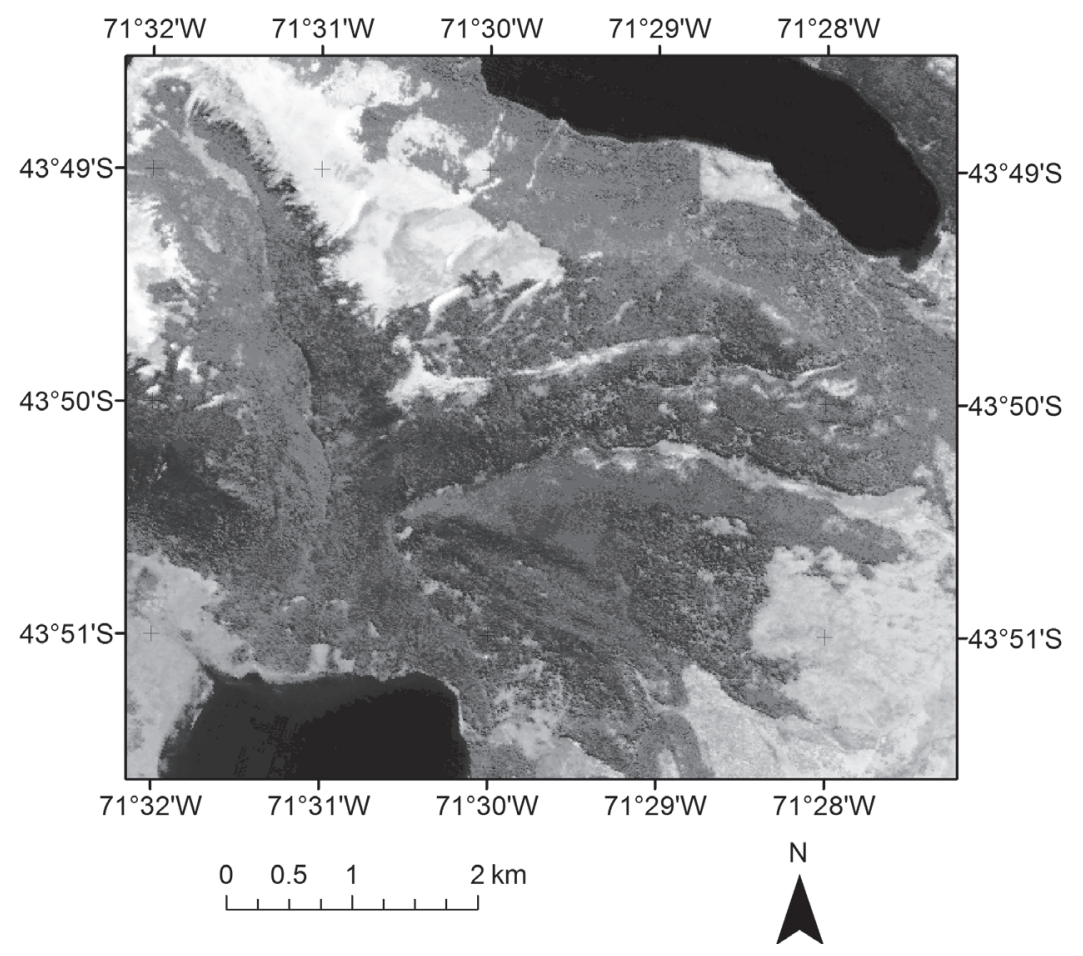

Fig. 1: Imagen SPOT-5 (banda del rojo) del área de estudio.

SPOT-5 (red band) of the study area. 
utilizado en numerosas aplicaciones relacionadas con teledetección, como el diseño de índices de vegetación y la estimación de parámetros biofísicos mediante inversión (Jacquemoud et al. 2006, Jacquemoud et al. 2009).

E1 PROSPECT simula la reflectividad y transmisividad a nivel de hoja, tiene su origen en el trabajo de Allen et al. (1969) y aún está en desarrollo (Feret et al. 2008). Sus variables de entrada son: el parámetro estructural de la hoja $(\mathrm{N})$, el contenido de clorofila $\mathrm{a}+\mathrm{b}(\mathrm{CCa}+\mathrm{b})$, el contenido de agua (CA) y el contenido de materia seca (CMS). El modelo SAIL (del inglés Scattering by Arbitrary Inclined Leaves) fue desarrollado por Verhoef (1984) y calcula la reflectividad direccional de un dosel sujeto a las simplificaciones vistas en el párrafo anterior. El dosel es determinado por el IAF, las características espectrales de las hojas y su distribución angular (LAD, del inglés Leaf Angle Distribution). Para calcular la reflectividad direccional, el SAIL tiene en cuenta la reflectividad del suelo y la geometría de iluminación. En resumen, el PROSAIL requiere como entrada a parámetros que caractericen la estructura-bioquímica de las hojas, la estructura del dosel y la geometría de observación e iluminación; ofreciendo como salida la reflectividad del dosel en una determinada dirección (la del sensor) y entre los 400 y $2500 \mathrm{~nm}$ de longitud de onda (Fig. 2).

Para resolver el PROSAIL se utilizó el software WinSAIL desarrollado por el Departamento de
Agricultura de los Estados Unidos (http://ars.usda.gov/ services/software). Para realizar la inversión se utilizó la técnica de tablas de consulta (LUT, del inglés Look-Up Table), que puede resumirse en tres pasos: (1) calcular el modelo variando los parámetros de entrada dentro de ciertos rangos e intervalos, (2) procesar cada resultado para ajustarlo a los datos satelitales con los que se invertirá, y (3) construir tablas donde se pueda consultar el valor de la entrada del modelo en función de la salida ajustada (Pragnere et al. 1999, Liang 2007, Jacquemoud 2009, Yebra \& Chuvieco 2009).

$\mathrm{Al}$ abordar el primer paso en la construcción de las tablas de consulta fue necesario establecer los mínimos y máximos esperados en los parámetros de entrada del PROSPECT, para lo que se recurrió a la bibliografía (Yebra \& Chuvieco 2008, Yebra \& Chuvieco 2009). El CMS de hojas de lenga fue reportado por Gómez et al. (1987) y Hertel et al. (2008), promediándose en 0.007 $\mathrm{g} \mathrm{cm}^{-2}$. Considerando que la forma y peso de las hojas del ñire son similares a las de la lenga (Gómez et al. 1987), se utilizó el CA de las hojas del ñire (JF Todone, datos no publicados), promediándose en $0.009 \mathrm{~g} \mathrm{~cm}^{-2}$. No se ha reportado el contenido de $\mathrm{CCa}+\mathrm{b}$ de la lenga o el ñire, por lo tanto, fue utilizado el promedio de 45 $\mu \mathrm{g} \mathrm{cm}^{-2}$ reportado para Fagus sylvatica L. por Le-Maire et al. (2008). Con respecto al parámetro N, la forma de obtenerlo es invirtiendo el modelo PROSPECT en condiciones de laboratorio (De Santis et al. 2006), lo que no ha sido realizado con especies patagónicas. Por lo
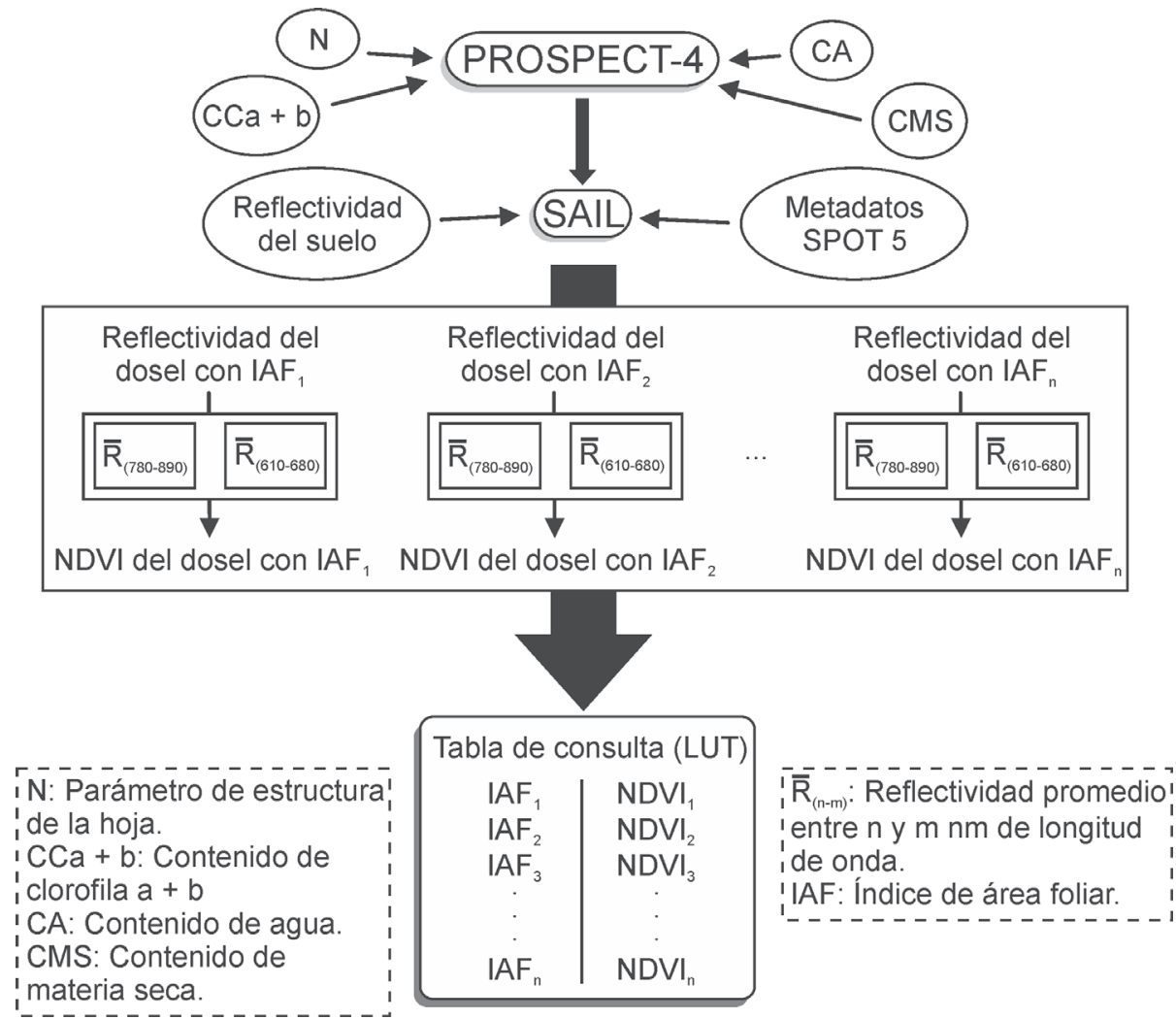

Fig. 2: Flujo metodológico para la generación de tablas de consulta (LUT) aplicables a la inversión del modelo PROSPECT + SAIL.

Methodology flow to generate look-up tables to invert the PROSPECT + SAIL model. 
tanto, se adoptó un $\mathrm{N}$ promedio de 1.7 en base al estudio de Le-Maire et al. (2008) con F. sylvatica.

Utilizar modelos físicos tiene como desventaja la complejidad de cálculo (Yebra \& Chuvieco 2008), por eso es que en este trabajo se propone utilizar un índice de vegetación para facilitar el proceso (Yebra et al. 2008b, Houborg et al. 2007). El IAF ha sido mayormente relacionado con el NDVI (Liang 2003, capítulo 8), el cual sintetiza el contraste entre la alta absorción a los $690 \mathrm{~nm}$ y la baja absorción a los $850 \mathrm{~nm}$, típico de la vegetación fotosintéticamente activa (Myneni et al. 1995) y atribuible a los pigmentos de la hoja y su estructura celular (Liang 2003, capítulo 3).

Se realizó un análisis de sensibilidad para evaluar cómo afecta al NDVI a nivel de hoja la exactitud en la determinación de los parámetros de entrada del PROSPECT. El NDVI a nivel de hoja fue calculado con la salida del PROSPECT, considerando el mismo intervalo del espectro electromagnético registrado por el SPOT-5 en el rojo e infrarrojo cercano. El análisis de sensibilidad consistió en variar un parámetro a la vez dentro de un rango mayor al mínimo y máximo esperado para la especie.

En base a los resultados del análisis de sensibilidad y los de Jing et al. (2004), se estableció que el IAF es sensible a la variación del $\mathrm{CCa}+\mathrm{b}$ (véase la discusión). Por lo tanto, se calculó el PROSAIL variando el CCa $+b$ dentro de un rango razonable para la especie y en intervalos que impliquen un nivel de variación detectable por la resolución radiométrica del sensor $(\mathrm{CCa}+\mathrm{b}$ de 20 a $70 \mu \mathrm{g} \mathrm{cm}^{-2}$ cada $5 \mu \mathrm{g} \mathrm{cm}^{-2}$ y IAF de 0.4 a 4 cada 0.2 ). De ese modo se finalizó con el primer paso para construir las tablas de consulta. Para el segundo paso, se calculó el NDVI (a nivel de dosel) con la salida del PROSAIL. Para el tercer paso, se construyó una tabla de consulta por cada $\mathrm{CCa}+\mathrm{b}$ probado, concretándose la inversión del PROSAIL. Sin embargo, se construyeron 11 tablas de consulta (una por cada $\mathrm{CCa}+\mathrm{b}$ ), pero solo una de ellas se corresponde con el $\mathrm{CCa}+\mathrm{b}$ adecuado para la lenga de la región (LUT óptima). Para identificar cuál es el $\mathrm{CCa}+\mathrm{b}$ adecuado, se validó el resultado de cada tabla de consulta (IAF estimado) con el IAF determinado en base a fotografías hemisféricas. Como la validación consistió en calcular el residuo medio cuadrático (RMS), la tabla de consulta con menor RMS fue considerada óptima (LUT óptima).

Con respecto a los parámetros de entrada del SAIL, la información referente a la geometría de observación e iluminación fue obtenida de los metadatos de la escena SPOT-5; de las LAD disponibles en el software WinSAIL, fue elegida la distribución planófila en base a observaciones de campo; la reflectividad del fondo fue calculada en base a la firma espectral de un suelo con 44 \% de arena, $39 \%$ de limo y $29 \%$ de arcilla (Fig. 2). Estos fueron los parámetros utilizados en todos los cálculos del SAIL.

\section{Métodos de estimación de área basal}

Para desarrollar los tres métodos de estimación de área basal evaluados en este trabajo (ED, E2P y FE2P), se utilizó la información de las 20 parcelas con datos de IAF. Las restantes 24 parcelas fueron utilizadas exclusivamente en el proceso de validación.

Con regresión por mínimos cuadrados se calcularon tres ecuaciones: (1) el IAF en función del NDVI, (2) el área basal en función del NDVI y (3) el área basal en función del IAF (Tabla 1). Combinando estas tres ecuaciones y el PROSAIL se establecieron los siguientes métodos de estimación de área basal (Fig. 3): estadístico directo (ED, ecuación 2); estadístico de dos pasos (E2P, ecuación 1 y 3) y físico-estadístico de dos pasos (FE2P, PROSAIL y ecuación 3 ).

El método ED es incluido como testigo de acuerdo con Labrecque et al. (2006) y Soenen et al. (2010). El E2P se basa en la propuesta de Hall et al. (2006), que en FE2P se modifica mediante el uso del modelo PROSAIL.

\section{Validación y análisis comparativo}

La exactitud de los métodos ED, E2P y FE2P (Fig. 3) fue calculada con 24 unidades de muestreo (n) a razón de seis por estrato. Estas unidades son independientes, esto es, ninguna de ellas fue utilizada en el ajuste estadístico de las ecuaciones o en la calibración del PROSAIL.

Los residuos (res) fueron calculados como la diferencia entre el valor observado $\left(\mathrm{V}_{\mathrm{obs}}\right)$ y el estimado $\left(\mathrm{V}_{\text {est }}\right)$; los residuos porcentuales fueron referidos al valor observado. La distribución normal de los residuos se verificó mediante la prueba de Shapiro Wilk. Se calculó la media y el desvío estándar (DE) de los residuos, además de las siguientes ecuaciones:

$$
\begin{gathered}
R M S=\sqrt{\frac{\sum r e s_{i}^{2}}{n}} \\
M e d i a_{|r e s|}(\%)=\frac{\sum|r e s(\%)|_{i}}{n}=\frac{\sum|r e s|_{i} \times 100}{\bar{V} \times n} \\
C V_{|r e s|}=\frac{D E_{|r e s|}}{M e d i a_{|r e s|}}
\end{gathered}
$$

TABLA 1

Ecuaciones de regresión $(n=20)$. RMS: residuo medio cuadrático; IAF: índice de área foliar; NDVI: índice de vegetación de diferencia normalizada; $\mathrm{AB}$ : área basal $\left(\mathrm{m}^{2} \mathrm{ha}^{-1}\right)$.

Regression equations $(n=20)$. RMS: root mean square; IAF: Leaf área index; NDVI: Normalized difference vegetation index; $A B$ : basal area $\left(\mathrm{m}^{2} \mathrm{ha}^{-1}\right)$.

\begin{tabular}{lcc}
\hline Variables & Ecuaciones & RMS del ajuste \\
\hline$(1) \mathrm{IAF}=\mathrm{f}(\mathrm{NDVI})$ & $\mathrm{IAF}=-9.669+13.550 * \mathrm{NDVI}$ & 0.27 \\
$(2) \mathrm{AB}=\mathrm{f}(\mathrm{NDVI})$ & $\mathrm{AB}=-143.551+242.895 * \mathrm{NDVI}$ & $12.13 \mathrm{~m}^{2} \mathrm{ha}^{-1}$ \\
$(3) \mathrm{AB}=\mathrm{f}(\mathrm{IAF})$ & $\mathrm{AB}=35.271 * \mathrm{IAF}$ & $13.27 \mathrm{~m}^{2} \mathrm{ha}^{-1}$ \\
\hline
\end{tabular}


siendo RMS: residuo medio cuadrático, |res|: residuo absoluto, V: media de la variable y CV: coeficiente de variación.

Para evaluar la significancia estadística de las diferencias en exactitud, se realizaron dos tipos de prueba de $\mathrm{t}$ de dos colas: contra cero (para evaluar tendencia) y de comparación de medias muestrales. Esta última, previa verificación de la igualdad de varianzas mediante una prueba de $\mathrm{F}$.

\section{RESULTADOS}

La relación entre los parámetros biofísicos y el área basal fue aproximadamente lineal (Figs. $4 \mathrm{~A}$ y $4 \mathrm{~B})$, por lo tanto, se utilizó regresión lineal para ajustar la ecuación 1 y 2 de la Tabla 1 . Para ajustar la ecuación 3 (Tabla 1), fue calculada una regresión lineal a través del origen a causa de la tendencia observada en la nube de puntos y la relación biológica esperada entre el IAF y el área basal, esto es, sin follaje no se espera encontrar área basal viva (Fig. 4C y Tabla 1).
En el análisis del modelo PROSPECT, las pendientes cercanas a cero de N-NDVI, CANDVI y CMS-NDVI (Fig. 5) evidencian que el NDVI a nivel de hoja fue solamente sensible a la variación del $\mathrm{CCa}+\mathrm{b}$.

La LUT óptima se corresponde con el $\mathrm{CCa}+$ $\mathrm{b}$ de $30 \mu \mathrm{g} \mathrm{cm}^{-2}$, ya que es el $\mathrm{CCa}+\mathrm{b}$ de menor RMS, como se demuestra en la Fig. 6. Por lo tanto, fue la tabla de consulta utilizada en el método FE2P.

El IAF medio $( \pm$ DE) de las parcelas de terreno fue de $1.57 \pm 0.52$ con $\mathrm{n}=20$. $\mathrm{El}$ área basal medio ( \pm DE) fue de $59.88 \pm 15.32 \mathrm{~m}^{2} \mathrm{ha}^{-1}$ con $\mathrm{n}=44$.

Para los tres métodos de estimación se confirmó que sus residuos tienen distribución normal con media significativamente diferente de cero (Tabla 2). El RMS, tanto en $\mathrm{m}^{2}$ ha-1 como en \%, indicó que el método E2P es el menos exacto, aunque en la Fig. 7 este se corresponde con la única distribución sin

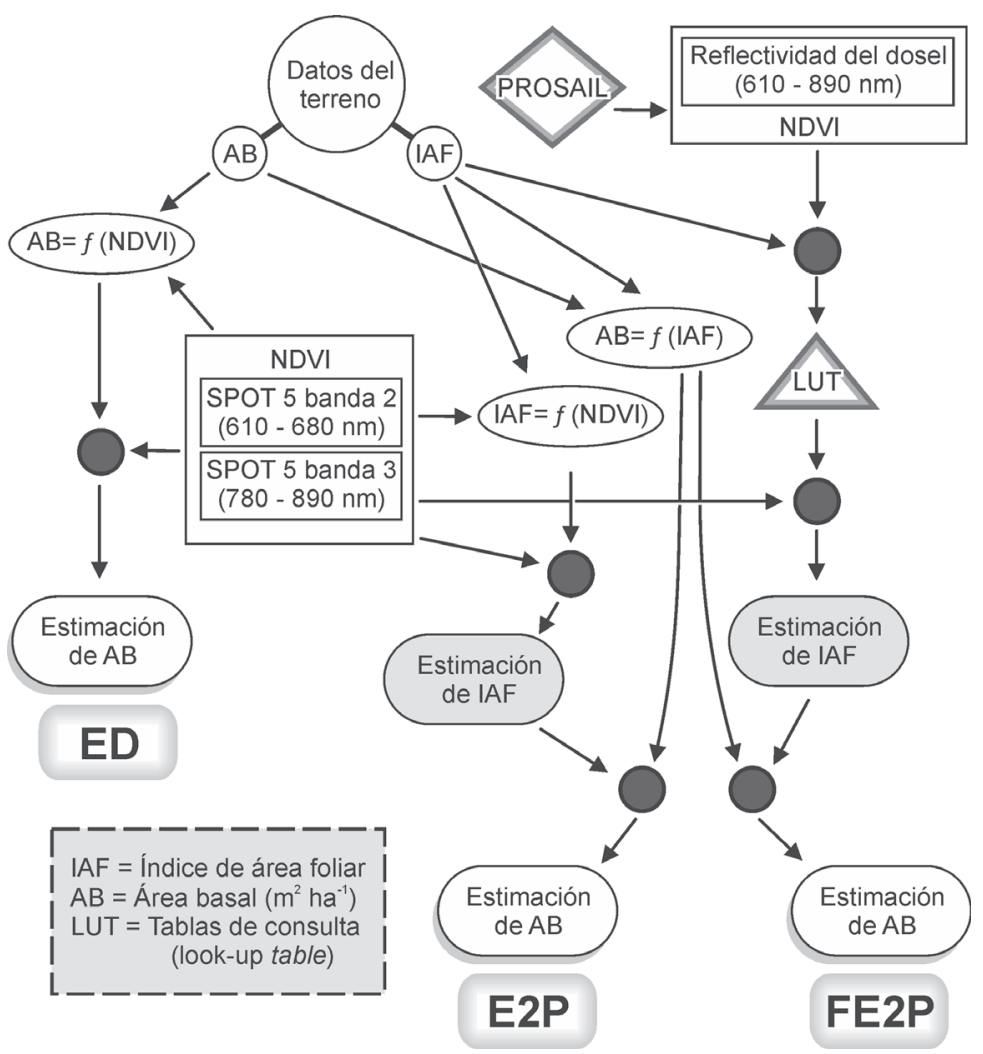

Fig. 3: Flujo metodológico de tres métodos alternativos para estimar área basal: estadístico directo (ED), estadístico de dos pasos (E2P) y físico-estadístico de dos pasos (FE2P).

Methodology flow of three alternative methods to estimate basal area: direct statistics (ED), two steps statistics (E2P) and two steps physics-statistics (FE2P). 
valores aislados. Sin embargo, la exactitud de los métodos no presenta diferencias significativas en la prueba de $t$ de dos colas realizada con los residuos expresados en $\mathrm{m}^{2}$ ha $^{-1}$ (ED vs. $\mathrm{E}^{2} \mathrm{P} \mathrm{t}_{46}=-1.541, \mathrm{P}=0.1301 ; \mathrm{ED}$ vs. FE2P $t_{46}=-1.046, \mathrm{P}=0.3009 ;$ E2P vs. FE2P t $\mathrm{t}_{46}=$ $-1.541, \mathrm{P}=0.1301)$.

\section{DISCUSIÓN}

La relación entre el NDVI a nivel de hoja y el CCa + b (Fig. 5) sigue una curva con crecimiento de tipo logarítmico. El NDVI a nivel de dosel y el $\mathrm{CCa}+\mathrm{b}$ también tienen una relación de tipo logarítmica, como lo
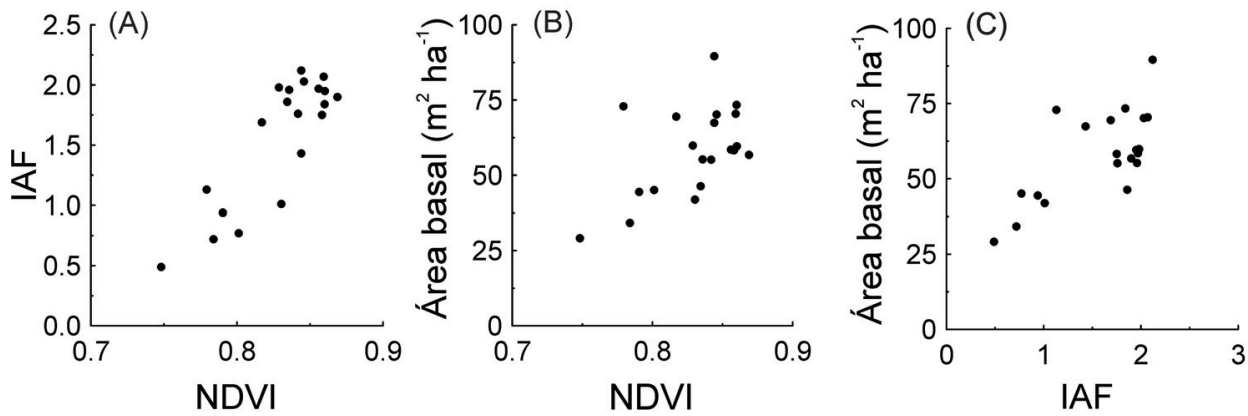

Fig. 4: Distribución de los datos utilizados para ajustar los modelos estadísticos y calibrar el modelo PROSPECT. Data distribution that was used to adjust the statistical model and calibrate the PROSPECT model.
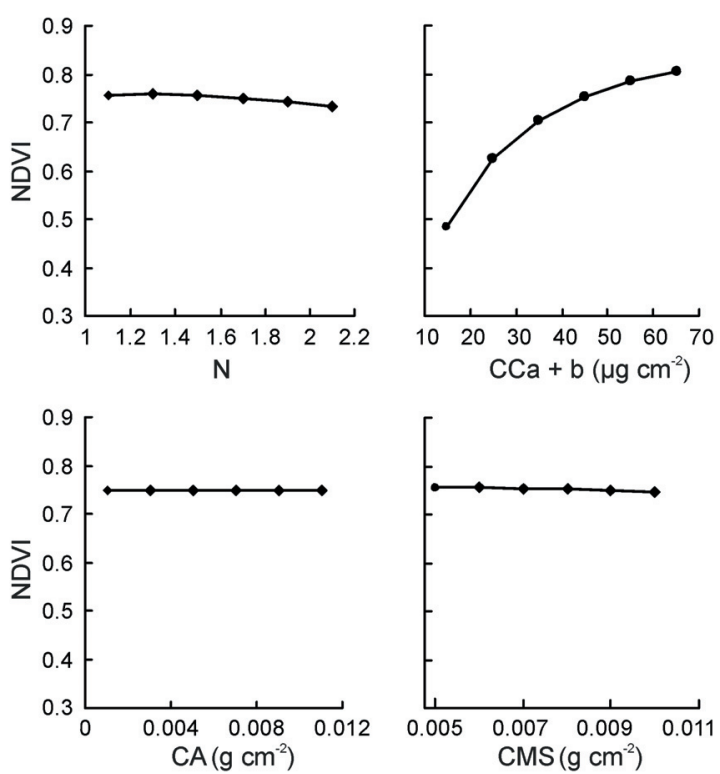

Fig. 5: NDVI a nivel de hoja calculado con la salida del modelo PROSPECT. En las abscisas, las variables de entrada: parámetro de estructura interna $(\mathrm{N})$, contenido de clorofila $\mathrm{a}+\mathrm{b}(\mathrm{CCa}+\mathrm{b})$, de agua $(\mathrm{CA})$ y de materia seca (CMS).

NDVI at leaf level calculated with PROSPECT model output. The abscissas are the model input variables: leaf structure parameter $(\mathrm{N})$, chlorophyll $\mathrm{a}+\mathrm{b}$ content $(\mathrm{CCa}+\mathrm{b})$, equivalent water thickness (CA) and dry matter content (CMS).

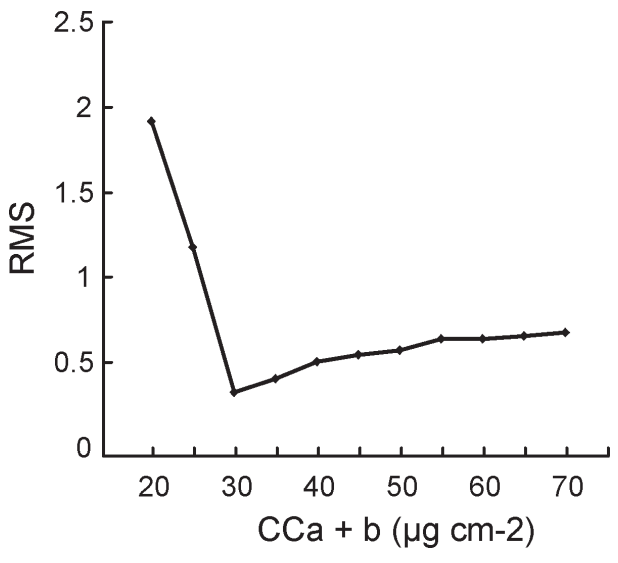

Fig. 6: Estimación de índice de área foliar mediante la inversión de los modelos PROSPECT y SAIL (PROSAIL). Residuo medio cuadrático (RMS) en función de la variación del contenido de clorofila $\mathrm{a}+\mathrm{b}(\mathrm{CCa}$ $+b)$.

Leaf area index estimation by model inversion technique applied to the couple PROSPECT and SAIL (PROSAIL). Root mean square error as a function of chlorophyll $\mathrm{a}+\mathrm{b}$ content $(\mathrm{CCa}+\mathrm{b})$ variation. 
demuestran Jing et al. (2004), quienes estudian la relación entre el NDVI a nivel de dosel y el $\mathrm{CCa}+\mathrm{b}$ para varios valores de IAF. La forma de la curva no varía sustancialmente al modificar el IAF, solamente cambia la ordenada al origen. Esto demuestra que la variación en reflectividad a nivel de hoja es proporcional a la calculada a nivel de dosel para el caso en que solamente se varíe el IAF.

Los resultados indican que la estimación de área basal mediante el método físico-estadístico implementado no difiere significativamente en exactitud con respecto a los métodos solo estadísticos (Tabla 2). Resultados similares fueron reportados por Soenen et al. (2010), quienes trabajaron con datos SPOT-5 y el método MFM (del inglés Multiple Forward Mode, basado en tablas de consulta) aplicado a la inversión del modelo GOMS (del inglés Geometric-Optical Mutual Shadowing) con el fin de estimar biomasa aérea de bosques de montaña. Los autores usan regresión con el NDVI como método testigo y, aunque no reportan los residuos porcentuales, reportan la media y el desvío estándar del residuo absoluto y la biomasa media calculada con datos del terreno. Con los datos publicados por Soenen

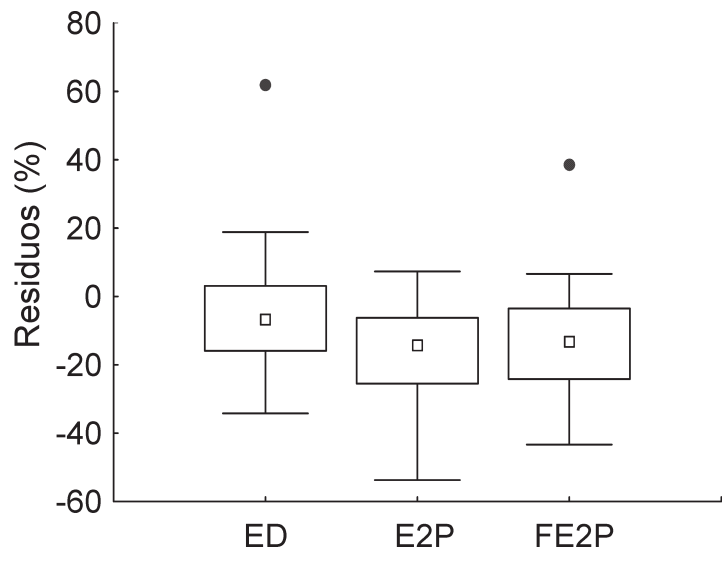

Fig. 7: Residuos porcentuales de tres métodos alternativos de estimación de área basal $(n=24)$. ED: estadístico directo; E2P: estadístico de dos pasos; y FE2P: físico-estadístico de dos pasos. Se Representa gráficamente la mediana $(\square)$, el rango intercuartil (caja), los valores aislados $(\bullet$, aquellos alejados de la mediana más de 1.5 veces el rango intercuartil) y el rango de valores no-aislados $(\mathrm{H})$.

Percentage residual of three alternative methods for basal area estimation $(n=24)$. ED: direct statistics; E2P: two steps statistics; and FE2P two steps physics-statistics. Median ( $\square$ ), interquartile range (box), outliers $(\bullet$, that are more than 1.5 times the interquartile range away from the median) and the no-outliers range $(H)$ have been graphically represented.

TABLA 2

Análisis de los residuos para validar tres métodos alternativos de estimación de área basal $(\mathrm{n}=24$ observaciones independientes). ED: Estadístico directo; E2P: Estadístico de dos pasos; y FE2P: Físico-estadístico de dos pasos; DE: Desvío estándar; RMS: Residuo medio cuadrático, |res|: Residuo absoluto, CV: Coeficiente de variación, SW-W: Prueba de normalidad de Shapiro Wilk; t: Prueba de t de dos colas contra cero.

Residual analysis to validate three alternatives methods of basal area estimation ( $\mathrm{n}=24$ independent observations). ED: Direct statistics; E2P: Two steps statistics; y FE2P: Two steps physics-statistics; DE: Standard deviation; RMS: Root mean square, |res|: Absolute residual, CV: Coefficient of variation, SW-W: Shapiro Wilk test; t: Two tailed t-test against zero.

\begin{tabular}{lccc}
\hline Estadísticos & ED & E2P & FE2P \\
\hline Media $\left(\mathrm{m}^{2} \mathrm{ha}^{-1}\right)$ & -5.53 & -9.95 & -8.62 \\
$\mathrm{DE}\left(\mathrm{m}^{2} \mathrm{ha}^{-1}\right)$ & 10.37 & 9.46 & 10.08 \\
RMS $\left(\mathrm{m}^{2} \mathrm{ha}^{-1}\right)$ & 12.67 & 14.89 & 14.35 \\
RMS (\%) & 19.17 & 22.56 & 20.09 \\
Media $_{\mid \text {res| }}(\%)$ & 14.27 & 18.12 & 16.44 \\
$\mathrm{CV}_{\mid \text {res| }}(\%)$ & 91.58 & 75.75 & 71.86 \\
SW-W & $-0.973 \mathrm{P}=0.7584$ & $0.948 \mathrm{P}=0.2538$ & $0.950 \mathrm{P}=0.2719$ \\
$\mathrm{t}_{23}$ & $-2.614 \mathrm{P}=0.0155$ & $-5.154 \mathrm{P}=0.0000$ & $-4.192 \mathrm{P}=0.0003$ \\
\hline
\end{tabular}


et al. (2010) se calculó la media [Media|res| (\%)] y el coeficiente de variación $\left(\mathrm{CV}_{\mid \text {res }}\right)$ de los residuos absolutos. Cuando estos autores usan el GOMS, la Media|res| es $21.13 \%$ con CV de 78 $\%(\mathrm{n}=36)$; cuando usan el modelo estadístico, es de $23.13 \%$ con CV de $89 \%(n=36)$.

De contrastar los resultados presentados en la Tabla 2 con los de Soenen et al. (2010) se desprende que los primeros son más exactos que los segundos y que en ambos casos la exactitud del modelo físico es similar a la del estadístico. Mientras que la mayor exactitud puede deberse solo a diferencias entre las áreas de estudio, la equivalencia en exactitud entre los métodos físicos y estadísticos puede ser de relevancia.

Yebra et al. (2008b) también encuentran similar exactitud entre la aproximación estadística y la física, pero en la estimación de contenido de humedad de pastizales con PROSAIL (CA y CMS) y con regresión estadística. En un trabajo posterior, con la hipótesis de que los modelos físicos son más robustos que los estadísticos, Yebra et al. (2008a) utilizan los modelos desarrollados por Yebra et al. (2008b) en dos áreas de estudio diferentes a aquella en que fueron ajustados. Los resultados indican que la exactitud del modelo físico es sustancialmente mayor a la del modelo estadístico, demostrando la hipótesis de robustez.

La mayor robustez de los modelos físicos permitiría desarrollar métodos automáticos para estimar parámetros biofísicos con teledetección satelital y sin datos del terreno, como sugiere Peddle et al. (2010). Los autores utilizan datos Landsat TM de un bosque de coníferas y aplican el mismo método que Soenen et al. (2010), pero sin calibrar previamente con datos de campo. Con los estadísticos de exactitud reportados por Peddle et al. (2010) [ $\mathrm{n}=4\left(2500 \mathrm{~m}^{2}\right.$ cada uno) ] fue posible calcular la Media|res| y el $\mathrm{CV}_{\text {|res| }}$. A continuación, estos se detallan para cada variable, respetando siempre el esquema "nombre de la variable, Media|res| $\left(\mathrm{CV}_{\mid \text {res }}\right)$ ": densidad del rodal, 10.2 (22) \%; radio horizontal de copa, 54.3 (10) \%; radio vertical de copa, 20.4 (15) \%; altura al centro de las copas, 25.6 (16) \%; altura de los árboles, 17.1 (16) \%; y distribución de alturas, 10.0 (39) \%.

El grado de exactitud alcanzado por Peddle et al. (2010) debe ser interpretado con reparo porque es sustancialmente superior al reportado por Soenen et al. (2010), en contra de lo esperado cuando no se utilizan datos del terreno. Eso podría explicarse por el bajo número de parcelas $(\mathrm{n}=4)$ utilizado por Peddle et al. (2010).

Aunque aún se deben realizar más estudios, los modelos físicos pueden ser clave para aplicar teledetección cuantitativa al monitoreo de bosques, principalmente debido a su robustez. La similitud en la exactitud del método FE2P en comparación con los estadísticos puros (Tabla 2) sugiere que el método de inversión aquí desarrollado es adecuado, siendo simple de implementar y posible de mejorar en la medida que se avance en el conocimiento ecofisiológico de la lenga. Además, señala la necesidad de futuras investigaciones para evaluar su robustez, de acuerdo con Yebra et al. (2008). Estas investigaciones consistirían en estimar el IAF de la lenga con el FE2P en otras áreas de estudio y con diferentes sensores remotos.

Para aplicar el modelo PROSAIL con la máxima eficiencia en los ecosistemas forestales de la Patagonia será necesario realizar determinaciones de contenido de clorofila, contenido de agua, materia seca e índice de área foliar de las principales especies. Asimismo, será necesario realizar la inversión del modelo PROSPECT en laboratorio con una muestra representativa de las hojas de cada una de estas especies, de acuerdo con De Santis et al. (2006) y Yebra \& Chuvieco (2009). Por otro lado, este estudio debe considerarse como un primer paso en la implementación de modelos físicos en ecosistemas forestales patagónicos, ya que existen modelos más complejos que deberían ser puestos a prueba en futuras investigaciones (Liang 2007). Si se desea mejorar el FE2P podría incorporarse la función de respuesta espectral del sensor (Liang 2003) en el segundo paso de la construcción de las tablas de consulta, pero esto sería a costa de incrementar la complejidad del cálculo. Finalmente, si bien el FE2P involucra al PROSAIL, aún depende de relaciones estadísticas, lo que implica una pérdida en la robustez del procedimiento. Esto señala la necesidad de investigar relaciones físico-estadístico-biológicas para desarrollar modelos que permitan relacionar parámetros biofísicos de primer orden con de segundo orden (variables que pueden obtenerse 
directamente de la inversión de modelos físicos con parámetros habitualmente medidos en los inventarios forestales). En ese sentido, un paso evidente es ampliar la base de datos con la que se realiza la relación estadística entre el IAF y el área basal.

AGRADECIMIENTOS: Este trabajo fue realizado en el marco del Proyecto de Investigación Científica y Tecnológica Orientada "Evaluación y Monitoreo de los Bosques Andino Patagónicos utilizando Técnicas de Percepción Remota Multisensor" (PICTO 36812), financiado por el Fondo para la Investigación Científica y Tecnológica (FONCYT) y cofinanciadores provinciales y nacionales de la Argentina. Se agradece al grupo de trabajo del Laboratorio de Geomática del CIEFAP y a Patricia Dankha e Isabel Ortega, representantes de la iniciativa Planet Action de Spot Image que colaboraron con los datos satelitales SPOT 5 (http://www.planet-action.org/web/85project-detail.php?projectID=4929).

\section{MATERIAL COMPLEMENTARIO}

E1 Material Complementario "Sobre el procesamiento de los datos satelitales: clasificación en estratos, generación de un modelo digital de elevación y corrección geométrico-radiométrica" se encuentra disponible online en http://rchn.biologiachile. cl/pdfs/2011/4/MC_Diaz_et_al_2011.pdf

\section{LITERATURA CITADA}

ALCOBÉ F (2008) Los bosques nativos de Argentina en el marco del proceso de reducción de emisiones derivadas de la deforestación y la degradación (REDD). Programa de las Naciones Unidas para el desarrollo: 6 pp. (en línea) URL: http://www. undp.org.ar/docs/prensa/brief-08-cambios.pdf (accedido Julio 5, 2010).

ALLEN WA, HW GAUSMAN, AJ RICHARDSON \& JR THOMAS (1969) Interaction of isotropic light with a compact plant leaf. Journal of the Optical Society of America 59: 1376-1379.

BAVA J \& PM LOPEZ-BERNAL (2005) Cortas de selección en grupo en bosque de lenga. Revista IDIA XXI: Forestales (Argentina) 8: 37-40.

BAVA J \& C RECHENE (2004) Dinámica de la regeneración de lenga (Nothofagus pumilio (Poepp. et Endl.) Krasser) como base para la aplicación de sistemas silvícolas. En: Arturo M, J Franji \& J Goya (eds) Ecología y manejo de los bosques: Capítulo 14 (CD-ROM). EDULP, La Plata.

BETTINGER P, J SIRY J, K BOSTON \& D GREBNER (2008) Forest management and planning. Academic Press de Elsevier, Amsterdam.

BRÉDA N (2003) Ground-based measurements of leaf area index: A review of methods, instruments and current controversies. Journal of experimental botany 54: 2403-2417.
BÖSWALD K, JD LENCINAS \& G LOGUERCIO (2002) Carbon reservoirs in temperate South American Nothofagus forests. TheScientificWorldJOURNAL 2: 53-75.

CHEN J (2002) Derivation and validation of Canada-wide coarse-resolution leaf area index maps using high-resolution satellite imagery and ground measurements. Remote Sensing of Environment 80: $165-184$.

CHEN JM \& TA BLACK (1992) Defining leaf area index for non-flat leaves. Plant, Cell and Environment 15: 421-429.

DE SANTIS A, P VAUGHAN \& E CHUVIECO (2006) Foliage moisture content estimation from one-dimensional and two-dimensional spectroradiometry for fire danger assessment. Journal of Geophysical Research 111: 1-12.

ECKERT S, T KELLENBERGER \& JD LENCINAS (2005) Classification and forest parameter extraction of patagonian lenga forests with aster and Landsat ETM+ data. Pecora 16 "Global Priorities in Land Remote Sensing”. Sioux Falls, South Dakota: 11 pp. (en línea) URL: http://www. asprs.org/publications/proceedings/pecora16/ Eckert_S.pdf (accedido Agosto 2, 2010).

EKLUNDH L, K HALL, H ERIKSSON, J ARDÖ \& P PILESJÖ (2003) Investigating the use of Landsat thematic mapper data for estimation of forest leaf area index in southern Sweden. Canadian Journal of Remote Sensing 29: 349-362.

FANG H, S LIANG, J TOWNSHEND \& R DICKINSON (2008) Spatially and temporally continuous LAI data sets based on an integrated filtering method: Examples from North America. Remote Sensing of Environment 112: 75-93.

FASSNACHT K (1997) Estimating the leaf area index of North Central Wisconsin forests using the Landsat Thematic Mapper. Remote Sensing of Environment 61: 229-245.

FASSNACHT K, W COHEN \& T SPIES (2006) Key issues in making and using satellite-based maps in ecology: A primer. Forest Ecology and Management 222: 167-181.

FERET J, C FRANÇOIS, G ASNER, A GITELSON, R MARTIN et al. (2008) PROSPECT-4 and 5: Advances in the leaf optical properties model separating photosynthetic pigments. Remote Sensing of Environment 112: 3030-3043.

FERNANDES R, J MILLER, J CHEN \& I RUBINSTEIN (2004) Evaluating image-based estimates of leaf area index in boreal conifer stands over a range of scales using high-resolution CASI imagery. Remote Sensing of Environment 89: 200-216.

FLORES FJ, HL ALLEN, HM CHESHIRE, JM DAVIS, M FUENTES et al.(2006) Using multispectral satellite imagery to estimate leaf area and response to silvicultural treatments in loblolly pine stands. Canadian Journal of Forest Research 36: 1587-1596.

GEMMELL F \& J VARJO (1999) Utility of reflectance model inversion versus two spectral indices for estimating biophysical characteristics in a boreal forest test site. Remote Sensing of Environment 68: 95-111.

GÓMEZ I, G GALLOPIN \& M GROSS (1987) Predicciones del peso seco y del área foliar en tres especies del género Nothofagus. Ecología (Argentina) 8: 69-80.

HALL RJ, DP DAVIDSON \& DR PEDDLE (2003a) Ground and remote estimation of leaf area index 
in Rocky Mountain forest stands, Kananaskis, Alberta. Canadian Journal of Remote Sensing 29: 411-427.

HALL RJ, RA FERNANDES, EH HOGG, JP BRANDT, C BUTSON et al. (2003b) Relating aspen defoliation to changes in leaf area derived from field and satellite remote sensing data. Canadian Journal of Remote Sensing 29: 299-313.

HALL RJ, R SKAKUN, E ARSENAULT \& B CASE (2006) Modeling forest stand structure attributes using Landsat ETM+ data: Application to mapping of aboveground biomass and stand volume. Forest Ecology and Management 225: 378-390.

HERTEL D, A THERBURG \& R VILLALBA (2008) Above- and below-ground response by Nothofagus pumilio to climatic conditions at the transition from the steppe-forest boundary to the alpine treeline in southern Patagonia, Argentina. Plant Ecology \& Diversity 1: 21-33.

HOUBORG R, H SOEGAARD \& E BOEGH (2007) Combining vegetation index and model inversion methods for the extraction of key vegetation biophysical parameters using Terra and Aqua MODIS reflectance data. Remote Sensing of Environment 106: 39-58.

JACQUEMOUD S, C BACOUR, H POILVÉ \& J FRANGI (2000) Comparison of four radiative transfer models to simulate plant canopies reflectance direct and inverse mode. Remote Sensing of Environment 74: 471-481.

JACQUEMOUD S, W VERHOEF, F BARET, P ZARCO-TEJADA, G ASNER et al. (2006) PROSPECT+SAIL: 15 years of use for land surface characterization. 2006 IEEE International Symposium on Geoscience and Remote Sensing 00: 1992-1995.

JACQUEMOUD S, W VERHOEF, F BARET, C BACOUR, PJ ZARCO-TEJADA, GP ASNER, C FRANCOIS \& S USTIN (2009) PROSPECT+SAIL models: A review of use for vegetation characterization. Remote Sensing of Environment 113: S56-S66.

JING J, L LIU, JIHUA WANG, JINDI WANG \& C ZHAO (2004) Uncertainty analysis for NDVI using the physical models. Proceedings of IEEE International Geoscience and Remote Sensing Symposium 1: 4321-4324.

JONCKHEERE I, K NACKAERTS, B MUYS \& P COPPIN (2005) Assessment of automatic gap fraction estimation of forests from digital hemispherical photography. Agricultural and Forest Meteorology 132: 96-114.

KANGAS A \& M MALTAMO (eds) (2006) Forest inventory: Methodology and applications. Springer, Dordrecht, Países Bajos.

LABRECQUE S, RA FOURNIER, JE LUTHER \& D PIERCEY (2006) A comparison of four methods to map biomass from Landsat TM and inventory data in western Newfoundland. Forest Ecology and Management 226: 129-144.

LANG A (1987) Simplified estimate of leaf area index from transmittance of the sun's beam. Agricultural and Forest Meteorology 41: 179-186.

LANG A (1991) Validity of surface area indices of Pinus radiata estimated from transmittance of the sun's beam. Agricultural and Forest Meteorology 57: 157-170.

LEE K, W COHEN, R KENNEDY, T MAIERSPERGER \& ST GOWER (2004) Hyperspectral versus multispectral data for estimating leaf area index in four different biomes. Remote Sensing of Environment 91: 508-520.

LE-MAIRE G, C FRANÇOIS, K SOUDANI, D BERVEILLER, J PONTAILLER et al. (2008) Calibration and validation of hyperspectral indices for the estimation of broadleaved forest leaf chlorophyll content, leaf mass per area, leaf area index and leaf canopy biomass. Remote Sensing of Environment 112: 3846-3864.

LENCINAS JD (2009) Bosques de montaña y teledetección óptica espacial: Desarrollo y perspectivas. Congreso Forestal Mundial: 18-23 (en línea) URL: http://www.cfm2009.org/es/ programapost/resumenes/uploads/bosques_de montana_y_teledeteccion_FD.pdf (accedido Julio $5,2010)$.

LIANG S (2003) Quantitative remote sensing of land surfaces. John Wiley \& Sons, Inc., Hoboken.

LIANG S (2007) Recent developments in estimating land surface biogeophysical variables from optical remote sensing. Progress in Physical Geography 31: 501-516.

MARTÍNEZ-PASTUR G (2000) Timber production of Nothofagus pumilio forests by a shelterwood system in Tierra del Fuego (Argentina). Forest Ecology and Management 134: 153-162.

MARTÍNEZ-PASTUR G, MV LENCINAS, PL PERI, JM CELLINI \& A MORETTO (2010) Investigación sobre manejo forestal a largo plazo en Patagonia Sur-Argentina: Lecciones del pasado, desafíos del presente. Revista Chilena de Historia Natural 83: 159-169.

MARTÍNEZ-PASTUR G, MV LENCINAS, PL PERI \& M ARENA (2007) Photosynthetic plasticity of Nothofagus pumilio seedlings to light intensity and soil moisture. Forest Ecology and Management 243: 274-282.

MERONI M, R COLOMBO \& C PANIGADA (2004) Inversion of a radiative transfer model with hyperspectral observations for LAI mapping in poplar plantations. Remote Sensing of Environment 92: 195-206.

MYNENI RB, FG HALL, PJ SELLERS \& AL MARSHAK (1995) The interpretation of spectral vegetation indexes. IEEE Transactions on Geoscience and Remote Sensing 33: 481-486.

NACIONES UNIDAS (2009) Informe de la Conferencia de las Partes sobre su $15^{\circ}$ período de sesiones, celebrado en Copenhague del 7 al 19 de diciembre de 2009 (Vol. 60566): 48 pp. (en línea) URL: http://unfccc.int/resource/docs/2009/cop15/ spa/11a01s.pdf\#page $=4$ (accedido Julio 7, 2010).

PEDDLE D, S BOON, A GLOVER \& F HALL (2010) Forest structure without ground data: Adaptive Full-Blind Multiple Forward-Mode reflectance model inversion in a mountain pine beetle damaged forest. International Journal of Remote Sensing 31: 2123-2128.

PELLETIER J, KR KIRBY \& C POTVIN (en prensa) Significance of carbon stock uncertainties on emission reductions from deforestation and forest degradation in developing countries. Forest Policy and Economics.

POULAIN M, M PENA, A SCHMIDT, H SCHMIDT \& A SCHULTE (2010) Relationships between forest variables and remote sensing data in a Nothofagus pumilio forest. Geocarto International 25: 25-43.

PRAGNERE A, F BARET, M WEISS, R MYNENI, Y KNYAZIKHIN \& LB WANG (1999) Comparison of three radiative transfer model inversion 
techniques to estimate canopy biophysical variables from remote sensing data. International Geoscience and Remote Sensing Symposium: 1093-1095 (en línea) URL: http:// ieeexplore.ieee.org/1pdocs/epic03/wrapper. htm?arnumber $=774543$ (accedido diciembre 15, 2010).

SAyDS (2005) Primer inventario nacional de bosques nativos. Secretaría de ambiente y desarrollo sustentable: $126 \mathrm{pp}$. (en línea) URL: http://www. ambiente.gov.ar/archivos/web/UMSEF/File/ PINBN/informe_nacional_pinbn.pdf (accedido Abril 22, 2010).

SHIN Y, M SEGUCHI, M KORIYAMA \& A ISNANSETYO (2010) Estimation of LAI in the forested watershed using ASTER data based on Price's model in summer and winter. European Journal of Forest Research 129: 1237-1245.

SOENEN SA, DR PEDDLE, RJ HALL, CA COBURN \& FG HALL (2010) Estimating aboveground forest biomass from canopy reflectance model inversion in mountainous terrain. Remote Sensing of Environment 114: 1325-1337.

SPOT IMAGE (2010) Niveles de preprocesamiento y precisión de localización. Spot Image: $4 \mathrm{pp}$. (en línea) URL: http://www.spotimage.com/ automne_modules_files/standard/public/p321_ aa993ee1add34ee97d993edf2108b6c5Niveles de preprocesamiento_ESP_sept2010.pdf (accedido julio 22, 2011).

SPRINTSIN M, A KARNIELI, P BERLINER, E ROTENBERG, D YAKIR \& S COHEN (2007) The effect of spatial resolution on the accuracy of leaf area index estimation for a forest planted in the desert transition zone. Remote Sensing of Environment 109: 416-428.

TAN B, M RAUTIAINEN, NV SHABANOV, S LINDER, Y KNYAZIKHIN et al. (2006) The importance of measurement errors for deriving accurate reference leaf area index maps for validation of moderate-resolution satellite LAI products. IEEE Transactions on Geoscience and Remote Sensing 44: 1866-1871.

THIMONIER A, I SEDIVY \& P SCHLEPPI (2010) Estimating leaf area index in different types of mature forest stands in Switzerland: A comparison of methods. European Journal of Forest Research 129: 543-562.

TRIMBLE (2001) Trimble survey controller: Reference manual. Trimble: 744 pp. (en línea) URL:
http://trl.trimble.com/docushare/dsweb/Get/ Document-7965/RefMan.pdf (accedido Julio 2, 2011)

VEBLEN TT (1996) Ecology of Southern chilean and argentinian Nothofagus forests. En: Veblen TT, R Hill \& J Read (eds) The ecology and biogeography of Nothofagus Forests: 293-353. Yale University press, New Haven, Londres.

VEBLEN TT, T KITZBERGER \& R VILLALBA (2004) Nuevos paradigmas en ecología y su influencia sobre el conocimiento de la dinámica de los bosques del sur de Argentina y Chile. En: Franji MJ \& J Goya (eds) Ecología y manejo de los bosques: Capítulo 12 (CD-ROM). EDULP, La Plata.

VERHOEF W (1984) Light scattering by leaf layers with application to canopy reflectance modeling: The SAIL model. Remote Sensing of Environment 16: 125-141.

WATSON D (1947) Comparative physiological studies in the growth of field crops. I: Variation in net assimilation rate and leaf area between species and varieties, and within and between years. Annals of Botany 11: 41-76.

WOLTER PT, PA TOWNSEND \& BR STURTEVANT (2009) Estimation of forest structural parameters using 5 and 10 meter SPOT-5 satellite data. Remote Sensing of Environment 113: 2019-2036.

YANG W, B TAN, D HUANG, M RAUTIAINEN, NV SHABANOV et al. (2006) MODIS leaf area index products: From validation to algorithm improvement. IEEE Transactions on Geoscience and Remote Sensing 44: 1885-1898.

YEBRA M \& E CHUVIECO (2008) Modelos de Simulación de Reflectividad en ecología: Potencialidades y problemas. Ecosistemas (España) 17: 23-38.

YEBRA M \& E CHUVIECO (2009) Generation of a species-specific look-up table for fuel moisture content assessment. IEEE Journal of Selected Topics in Applied Earth Observations and Remote Sensing 2: 21-26.

YEBRA M, E CHUVIECO \& I AGUADO (2008a) Comparación de modelos empíricos y de transferencia radiativa para estimar contenido de humedad en pastizales: poder de generalización. Revista de Teledetección (España) 29: 73-90.

YEBRA M, E CHUVIECO \& D RIAÑNO (2008b) Estimation of live fuel moisture content from MODIS images for fire risk assessment. Agricultural and Forest Meteorology 148: 523-536. 


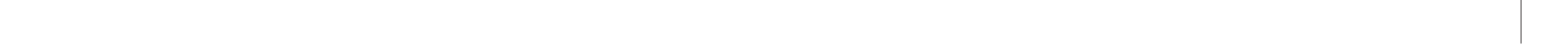

